\title{
Supporting QoS with Location Aware Pre-reservation in Mobile Ad Hoc Networks
}

\author{
Xiang Chen, Wei Liu, Yuguang Fang \\ Dept. of Electrical and Computer Engineering \\ University of Florida \\ Gainesville, FL 32611, U.S.A. \\ \{xchen@ecel., liuw@, fang@ece.\}ufl.edu
}

\author{
Maria C. Yuang \\ Dept. of Computer Science and Information Engineering \\ National Chiao Tung University \\ Hsinchu, Taiwan \\ mcyuang@csie.nctu.edu.tw
}

\begin{abstract}
Due to MAC collisions and mobility, quality of service (QoS) provisioning in mobile ad hoc networks is very challenging. In this paper, we propose an innovative scheme to grant high priority to communications between some important nodes in TDMA based ad hoc networks. In addition to bandwidth reservation in previous QoS routing approaches, our scheme adopts a new approach: bandwidth pre-reservation. Some bandwidth is pre-reserved at intermediate nodes in a quadrangleshaped area formed between important nodes. The key idea is to utilize each node's geographic location information and minimize potential scheduling conflicts for transmissions. In this way, time slot collisions in adjacent wireless links along a path can be reduced so that more high priority connections can be accepted into the network. Extensive simulations show that our scheme can successfully provide better communication quality at a relatively low price.
\end{abstract}

\section{INTRODUCTION}

There is a growing need to provide quality of service (QoS) in wireless mobile ad hoc networks, as they are finding more applications in many fields such as battlefield communications, disaster rescue, and inimical environment monitoring. Due to the lack of fixed wired infrastructure in ad hoc networks, all the communications between wireless mobile nodes are conducted over bandwidth limited and error-prone wireless links, with intermediate nodes acting as routers in between the source and destination. Therefore, it is very difficult for ad hoc networks to support QoS for real-time services or some connection-oriented applications.

Various schemes have been proposed to address this challenging problem at both layers. On medium access control (MAC) protocol designs, much work has been done to support QoS. In [1][2], improvements based on random access MAC protocol such as the IEEE 802.11 were proposed. At the same time, various techniques were explored on the side of Time Division Multiple Access (TDMA) MAC [3][4].

At network layer, a variety of QoS routing schemes have been proposed, with the primary goal to find a path from the source to the destination that satisfies the desired QoS

The work of Chen, Liu and Fang was supported in part by the U.S. National Science Foundation under Faculty Early Career Development Award ANIR0093241 and under grant ANI-0220287. The work of Yuang was supported in part by the National Science Council, Taiwan, under Grant NSC 92-2213-E-009-115. requirement. In [5] Lin and Liu proposed a scheme to calculate the end-to-end bandwidth of a path under a CDMA-overTDMA mechanism. Zhu and Corson proposed an efficient algorithm called forward algorithm for calculating the end-toend bandwidth on a path and reserving required bandwidth so that a QoS route could be established [6]. In [7], a framework of reliable routing was proposed by placing some reliable nodes in some special places in the network. Location information has been leveraged to reduce route discovery overhead [8] [9] or to scale to large and dense wireless ad hoc networks [10].

In addition to providing QoS, the network needs to provide service differentiation. For instance, in battlefield, the communication requests among some army commanders should be always accepted with higher priority compared with the communications between soldiers. Another typical scenario is that in cluster based hierarchical ad hoc networks, connections among cluster heads should be preferentially treated. In such cases, communications between such important nodes (like commanders or cluster heads, henceforth called $i$ nodes) should be granted higher priority as well as QoS guarantee.

Since traditional QoS routing scheme cannot provide a good solution to this, we propose a scheme based on cooperation of both the routing layer and the MAC. It is able to grant higher priority to connections between any two i-nodes (henceforth called i-connections) with QoS guarantees in a TDMA based ad hoc networks. By utilizing each node's geographic location information and forming a quadrangleshaped area, this scheme pre-reserves bandwidth along the possible paths connecting any two i-nodes, and reserves bandwidth for accepted QoS connections in such a way that collisions of time slots in time slotted MAC layer are significantly reduced. As a result, the network bandwidth is used more efficiently and the connection blocking probability for i-connection is greatly reduced, without seriously hurting admissions of other connections. To the best of our knowledge, this is the first paper to improve the performance of traditional QoS routing schemes in TDMA networks with routing layer bandwidth pre-reservation and MAC layer collision resolution.

The remainder of this paper is organized as follows. In Section II, the system model is introduced. The details of our bandwidth pre-reservation are presented in Section III. In 
Section IV, performance evaluation is given with discussion. Finally, concluding remarks are given in the Section V.

\section{SYSTEM MODEL}

We consider a mobile ad hoc network consisting of $N$ nodes, among which there are $M$ i-nodes. The system adopts TDMA as its channel access mechanism. As in [5], the transmission time scale is divided into frames, each of which contains a fixed number of time slots. Each frame is comprised of two phases. One is control phase and the other is data phase. In the control phase, all kinds of control functions are performed, such as pre-reservation request propagation, bandwidth reservation and connection setup, frame and slot synchronization. Each node may use a predefined slot to broadcast control messages to all of its neighbors. Data phase is used to transmit and receive data packets. For each time slot used to transmit or receive data (called data slot), it may be in one of the four states at one particular time: FREE, PRE RESERVED, USED_TX and USED_RX. They indicate the state of the data slot being free, pre-reserved, used to transmit or receive, respectively. All connections considered in this paper are connection-oriented and requires constant bandwidth. In ad hoc networks using TDMA, bandwidth is measured in terms of time slots. Hereafter, the terms bandwidth and time slot are used interchangeably. A connection will specify its QoS requirement as the number of transmission time slots it needs prior to being admitted into the network. In the network, in addition to i-connections whose both endpoints are i-nodes, all the other connections are ordinary connections.

We assume in this work that each node is half-duplex, i.e., it cannot transmit and receive simultaneously. To successfully transmit a packet, both the transmitter and receiver nodes need to have one or more common time slots. We also assume that each node is aware of its own geographic location and time is synchronized at each node, as current Global Positioning System (GPS) [11] can provide accurate location information and global timing. Finally, it is assumed that all i-nodes are aware of each other's geographic location. Since i-nodes need to frequently communicate with each other, they can piggyback their own location information and mobility information such as velocity and direction in data packets. I-nodes other than the source and the destination of $i$-connections can also acquire this information by overhearing.

\section{LOCATION-AWARE BANDWIDTH PRE-RESERVATION}

To reduce the blocking probability of i-connections, we need to purposely pre-reserve some network resources for them beforehand. To distinguish from normal bandwidth reservation, hereafter, we call it bandwidth pre-reservation.

\section{A. Pre-Reservation Requests}

To achieve efficiency, the bandwidth needs to be dynamically pre-reserved. Therefore, a propagation and processing mechanism for pre-reservation requests is introduced to inform each node when to pre-reserve bandwidth, how much bandwidth to pre-reserve for future incoming iconnection requests, and when to do de-pre-reservation.
As shown in Fig. 1, in order to pre-reserve some bandwidth along the path to i-node $j$, i-node $i$ broadcasts a message called pre-reservation request to its neighbors, such as node $n$. The request contains the following information: a) the preferred number of time slots to be pre-reserved, num_req; b) the duration, timer, for which the time slots may be pre-reserved; c) the location of both i-node $i$ and i-node $j$; d) i-node $i$ and $j$ 's identities (IDs); e) a unique sequence number. Upon reception of the request, each one-hop neighbor of i-node $i$, like node $n$, will retrieve the location information of the source and the destination i-node. With this information, a quadrangle-shaped influence area will be built as shown in Fig. 1. The angle $\alpha$ adjusts the size of influence area and hence the control overhead incurred. If the neighbor happens to fall into this area, like node $n$, it will try to pre-reserve the number of time slots as required. Assume the pre-reservation upper bound at node $n$ is $U B$, and the current number of pre-reserved time slots is num_tot. Then, node $n$ will take one of the following actions:

1) num_tot + num_req $<=U B$, which means the node can pre-reserve up to num_req free time slots. Then the node will choose either num_req time slots or all currently free time slots denoted by num free, whichever is smaller, and tag them with flag PRE_RESERVED. Then num_tot $=$ num_tot + min(num_req, num_free). The criterion governing how to choose which time slot to tag will be described in the following subsection. Besides, each chosen time slot will be associated with a timer, slot_timer( $k$ ), where $k$ is the ID of the time slot. Initially, slot_timer $(k)$ is set to 0. slot_timer $(k)=\operatorname{timer}$. When slot_timer(k) expires and the time slot will be freed, i.e., marked with flag FREE again.

2) $n u m$ tot $=U B$, which means no more free time slots can be pre-reserved. Then the node will rank all the pre-reserved time slots in an increasing order of the value of their attached timer. The first num_req time slots' timer will be increased by a value of timer, i.e., slot timer $(k)=$ slot timer $(k)+$ timer, with the flag PRE RESERVED unchanged. By doing this, though the number of pre-reserved time slots does not increase, their pre-reservation time is prolonged. Thus, this has the same effect as increasing the pre-reserved time slot number.

3) num_tot $>U B$. Note that this may occur since we dynamically adjust $U B$. In this case, the node will choose the first $U B$ time slots with the largest timer value. Among the $U B$ time slots, num tot time slots with the least timer value will be increased by a value of timer. In this way, the rest $U B$ num_tot time slots will be left without updating, which will thus expire soon.

4) num_tot $<U B$ and num_tot + num_req $>U B$. Then the node will choose $(U B-$ num_tot $)$ free time slots and prereserve them as described in 1$)$. The left (num_req - (UB num_tot )) time slots will be processed according to 2). Then num_tot $=U B$.

Next, node $n$ will broadcast the pre-reservation request message to its neighbors. In Fig. 1 , they are node $n+1, n+5$. Then, node $n+1$ and node $n+5$ will also check their position. If they are also in this quadrangle, such as node $n+1$, they will take the actions mentioned above. If they are not, such as node $n+5$, they simply ignore the request. So node $n+5$ will not pass on the pre-reservation request to node $n+6$. To prevent a node 
from pre-reserving more than once for the same pre-reservation request, repeated reception of the same request will be detected by source and destination i-node's ID and the sequence number.

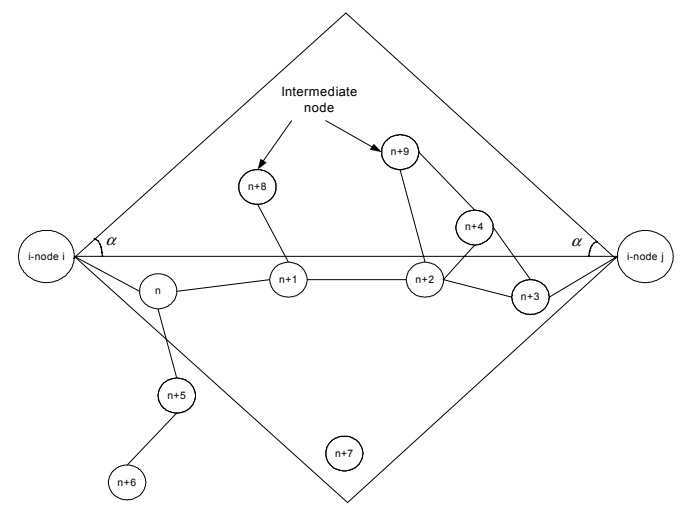

Figure 1. Illustration of a quadrangle-shaped influence area

The frequency of sending out pre-reservation request is adjusted by a timer, TIMER $R_{\text {update }}$, maintained at each i-node. Whenever this timer expires, the i-node will send another prereservation request and reset it. At the first glance, the overhead can be reduced by increasing TIMER $R_{\text {update }}$. However, with large $T I M E R_{\text {update }}$, bandwidth pre-reservation cannot quickly respond to the network topology change, which will cause performance degradation. Thus, TIMER $R_{\text {update }}$ should be set such that a good balance between overhead and performance can be achieved.

In this way, we clearly see that the pre-reservation at each intermediate node is only done when the node resides in the influence area and released when the node moves out. Therefore, this mechanism suits well with the mobile nature of ad hoc networks. Also, it is robust against transmission errors of pre-reservation requests, since upcoming pre-reservation requests will replace the previously lost or corrupted requests. It is important to note that the pre-reserved time slots are not explicitly tied to the use of any two specific i-nodes, though they may be pre-reserved upon their requests. As a result, every i-connection can make use of them if they are available, and statistical multiplexing gain could be obtained.

\section{B. Bandwidth Pre-Reservation Criterion}

In ad hoc networks, time slots belonging to neighboring wireless links within one-hop and two-hop range may collide with each other. As a result, even though pre-reservation can set aside some free time slots at each node for i-connections, these slots, if carelessly pre-reserved, may be useless because of collisions. Intuitively, if a wireless link can be isolated from the interference due to its neighboring links, more time slots at the link can be used due to the lack of collision. Motivated by this intuition, we propose the following scheme to pre-reserve time slots, which achieves interference isolation by taking advantage of each node's location information.

Assume the network geographically occupies a rectangular area with the size of $X \times Y \mathrm{~m}^{2}$. If the area is not a regular rectangle, it can be approximated with the smallest rectangle which can cover all the entire area. The transmission range of each node is $R \mathrm{~m}$. A grid structure is built by dividing the network area into a grid of cells with size $2 R \times 2 R$. As a result, along the horizontal axis, there are $I=X / 2 R$ cells. And along the vertical axis, there are $J=Y / 2 R$ cells. The cells are denoted by $(i, j)$, as shown in Fig. 2 .

Next, time slots in a frame are also divided. Assume there are $S$ data slots in one time frame. They are divided into two parts, denoted by $H_{1}$ and $H_{2}$, respectively. $H_{1}$ is the set of time slots which may be pre-reserved for connections along the horizontal direction and $\mathrm{H}_{2}$ is the counterpart for connections along vertical direction. To simplify notation, we also use $H_{1}$ and $H_{2}$ to refer to their respective size. The partition is in proportion to the dimension of the geographic area, i.e.,

$$
\begin{aligned}
& H_{1}=\left\lfloor\frac{I}{I+J} S\right\rfloor \\
& H_{2}=\left\lfloor\frac{J}{I+J} S\right\rfloor
\end{aligned}
$$

As shown in Fig. 2, the time slots in $H_{l}$ are further equally divided into $K_{h}\left(K_{h}>=2\right)$ sections, i.e., section $0,1,2, \ldots, K_{h}-1$. Similarly, $H_{2}$ are further equally divided into $K_{v}\left(K_{v}>=2\right)$ sections. Note that there is some overlap, $S_{o}$, between every two adjacent sections, which allows for the cases where some wireless links may span the boundary of two adjacent cells. In other words, one end of the link is located in one cell while the other end is located in an adjacent cell. Next, the geometrical position of each cell is mapped to the time axis. Along the horizontal direction, cell $(i, j)$ is mapped to time slot section $\bmod \left(j, K_{h}\right)$. Along the vertical direction, cell $(i, j)$ is mapped to time $\operatorname{slot} \operatorname{section} \bmod \left(i, K_{v}\right)$. Function $\bmod (p, q)$ returns the modulus obtained by dividing $p$ into $q$.

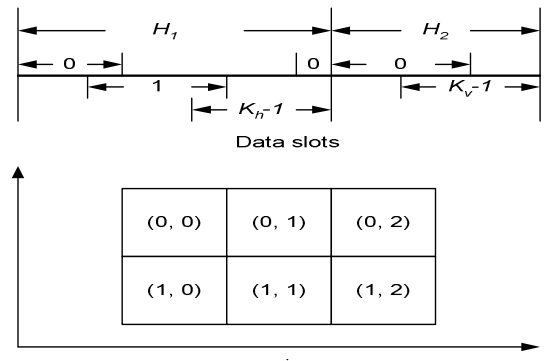

Geographical location

Figure 2. Location and time division

With such mapping, we show how time slots are prereserved so that collision could be significantly reduced. Suppose after processing a pre-reservation request, node $n$, located in cell $(i, j)$, should pre-reserve bw_num $(<=$ num_req) time slots. It will choose from its corresponding time sections according to its location.

Note that in general, the pre-reserved time slots chosen from different sections will not overlap. Any three consecutive hops are thus very likely to use different time slots, as they may span one single cell. As a result, the probability that they will collide with each other is greatly reduced, although the available bandwidth in each hop may is still the same. So when the QoS routing protocol is making bandwidth reservation 
along the path, it will find a path with sufficient bandwidth with a larger probability.

\section{Upper Bound of Bandwidth to Be Pre-reserved}

The upper bound, $U B$, is used to ensure that bandwidth is not excessively pre-reserved, which may cause bandwidth underutilization. Meanwhile, it provides the tradeoff between the performances of these two types of connections. To adapt to instantaneous network dynamics, such as traffic arrival information, node's geographic location, and node degree, it must be dynamically adjusted.

Each node in the network will monitor the incoming prereservation requests and the bandwidth requirement of each request. Let $T I M E R_{\text {avg }}$ be the average of the timers associated with the pre-reserved time slots, and let $\lambda_{p}$ be the arrival rate of the pre-reservation requests observed at a node. Besides, denote the average bandwidth requirement of each pre-reservation request by $B W$. Then we may set the upper bound to the following:

$$
U B=T I M E R_{\text {avg }} \lambda_{p} B W \Delta
$$

where $\Delta(0<\Delta<1)$ is a design parameter introduced to keep $U B$ from becoming too large, and $\lambda_{p}$ can be estimated using exponential moving average.

\section{Modifications to QoS Routing}

To enable i-connections to utilize the pre-reserved bandwidth, we need to change the QoS routing protocol accordingly. When the QoS routing protocol searches for path bandwidth, it cannot reserve the pre-reserved time slots for ordinary connections, although it can reserve either free or prereserved time slots for i-connections. Since our bandwidth prereservation scheme ensures that each node' pre-reserved time slots are spread over time axis according to its location, more time slots can be used by i-connections, which in turn enjoys a higher admission rate than ordinary connections. In this paper, we modified the Forward Algorithm (FA) [6] to calculate path bandwidth.

\section{PERFormance EVAluAtion}

Our simulation study is carried out using OPNET Modeler 8.0. An ad hoc network consisting of 80 mobile nodes is simulated in a $1600 \times 400 \mathrm{~m}^{2}$ area. The initial position of each mobile node is uniformly distributed in the entire network. The waypoint mobility model is used. There are 8 i-nodes, i.e., $10 \%$ of all nodes. Connection requests arrive following Poisson process, each requiring one data slot in each frame. The duration of each connection is exponentially distributed with mean of 60 seconds. In each time frame, the data slot is $5 \mathrm{~ms}$ and the control slot is $0.1 \mathrm{~ms}$. There are 30 control slots in control phase and 72 data slots in data phase, so the frame length is $30 * 0.1+72 * 5=363 \mathrm{~ms}$. We assume one data packet can be transmitted in one data slot. The angle $\alpha$ is 45 degree unless otherwise stated. The simulation duration is 900 seconds. Three schemes are compared. The first one is the scheme without any pre-reservation. The second is our proposed scheme, which makes bandwidth pre-reservation in a quadrangle area for i-connections. The last one also adopts bandwidth pre-reservation. However, it only pre-reserves time slots along the shortest path between any two i-nodes. In the figures presented below, these three are denoted by No prereservation, Quadrangle, and $S P$, respectively.

Fig. 3 shows the performances of the three schemes in terms of connection blocking probability. For i-connection, our scheme provides the lowest blocking probability among all three schemes. This is expected as each i-node is sending prereservation requests to pre-reserve some time slots at the intermediate nodes within the influence area. Scheme $S P$ is worse since it only pre-reserves slots at nodes on the shortest path, which may be unavailable due to mobility. We also observe that as the mobility is increased, the connection blocking probability is slightly reduced. This is not unexpected considering the following fact. When mobility increases, the ongoing connection may be dropped, releasing the bandwidth occupied for newly incoming connections. Therefore, the connection blocking probability drops instead of increasing. For ordinary connections, the performance of scheme No prereservation is the best. This is reasonable since the entire network bandwidth is fixed, as more bandwidth is given to iconnection, less bandwidth is left for accommodating ordinary connections. However, the differences are very small.

Next, we investigate the performances of these three schemes when re-routing is considered. Fig. 4 presents the rerouting successful probabilities for both types of connections. Again, for i-connections, our scheme, Quadrangle shows the best performance, since it pre-reserves bandwidth in the quadrangle. When one connection is dropped due to mobility, it will have high probability to find another path with sufficient bandwidth in the quadrangle. SP is worse and No prereservation is worst. For ordinary connections, their performances are in an opposite order.

Fig. 5 illustrates the throughput performance in terms of successfully received packets. For all three schemes, throughput is close, increasing as mobility decreases. This shows that although our scheme adopts bandwidth prereservation for i-connection, the network throughput is not seriously degraded.
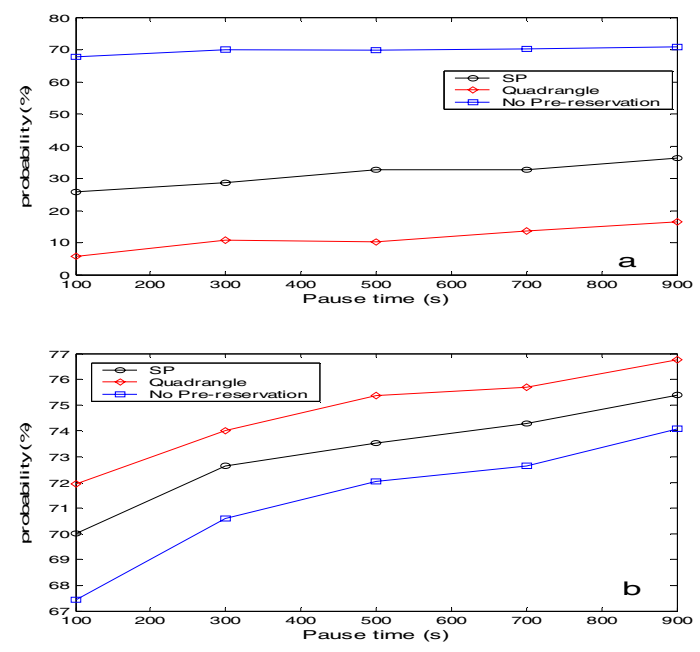

Figure 3. Connection blocking probability. (a) i-connection (b) ordinary connection 
The number of pre-reserved time slots in the network is presented in Fig. 6. As expected, our scheme pre-reserves more time slots compared with the other two. However, considering the entire network has a number of time slot $72 * 80=5760$, we only need to pre-reserve about $2.4 \%$. Fig. 7 shows the control overhead incurred by sending pre-reservation requests. We count the overhead as the number of times for which each node, either i-nodes or ordinary nodes, broadcasts the prereservation request. We see that the overhead is small. With the lowest blocking probability and highest re-routing successful probability for i-connections and the small control overhead incurred, our scheme, i.e., scheme Quadrangle is able to provide high priority communication service to i-nodes with QoS guarantee at a low cost. Scheme SP seems to perform fairly well, however, it cannot be used with some on-demand routing protocols as the shortest path information is not existent when doing bandwidth pre-reservation.

\section{CONCLUSION}

In this paper, we proposed a novel cross-layer approach for QoS provisioning and service differentiation in TDMA based ad hoc networks. By using geographic location information, we can pre-reserve time slots at intermediate nodes in a quadrangle-shaped area between important nodes. In this way, this scheme can not only reduce the transmission collision in adjacent wireless links and hence increasing spatial reuse, but also adapt to network topology change due to mobility. Therefore, more bandwidth can be used to provide high priority communication services to some important nodes, a goal that traditional QoS routing schemes cannot achieve without collaboration with lower layers. Extensive simulation results show that our scheme is able to achieve this goal with small overhead.
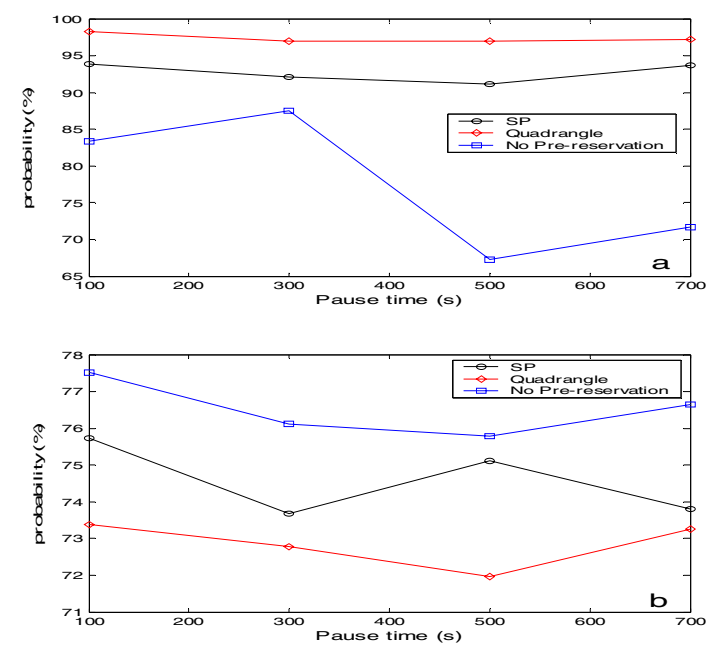

Figure 4. Re-routing successful probability. (a) i-connection (b) ordinary connection

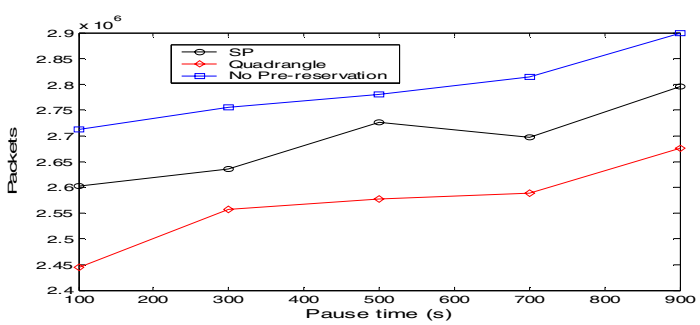

Figure 5. Throughput

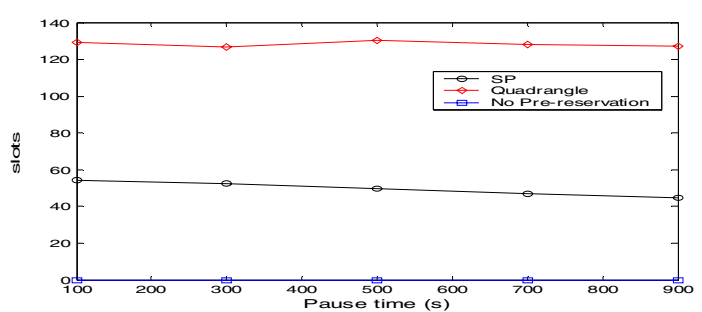

Figure 6. Average number of pre-reserved time slots

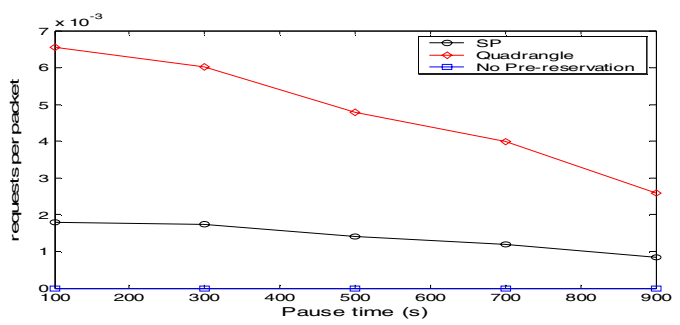

Figure 7. Pre-reservation requests per received packet

\section{REFERENCES}

[1] G-S Ahn, A. T. Campbell, A. Veres and L-H Sun, "SWAN: service differentiation in stateless wireless ad hoc networks", IEEE INFOCOM, 2002

[2] V. Kanodia, C. Li, A. Sabharwal, B. Sadeghi and E. Knightly, "Distributed Multi-hop scheduling and medium access with delay and throughput constraints", $A C M$ MOBICOM'01

[3] I. Chlamtac, A. Farago and H. Zhang, "Time-spread multiple-access (TSMA) protocols for multihop mobile radio networks", IEEE/ACM Transactions on Networking, vol. 5, pp. 804-812, Oct. 1997.

[4] A-M. Chou, V. O. K. Li, "Slot allocation strategies for TDMA protocols in multihop packet radio networks", IEEE INFOCOM, 1992.

[5] C. R. Lin and J. -S. Liu, "QoS routing in ad hoc wireless networks," IEEE JSAC, vol. 17, pp. 1426-1438, Aug. 1999.

[6] C. Zhu and M. S. Corson, "QoS routing for mobile ad hoc networks", IEEE INFOCOM, 2002.

[7] Z. Ye, S. V. Krishnamurthy and S. K. Tripathi, "A framework for reliable routing in mobile ad hoc networks", IEEE INFOCOM, 2003.

[8] Y. Ko and N. Vaidya, "Location-aided routing (LAR) in mobile ad hoc networks", ACM/IEEE MobiCom 1998.

[9] R. Banka and G. Xue, "Angle routing protocol: location aided routing for mobile ad-hoc networks using dynamic angle selection", IEEE MILCOM 2002.

[10] B. Karp and H. Kung, "GPSR: Greedy perimeter stateless routing for wireless networks", ACM/IEEE MobiCom 2000.

[11] B. W. Parkinson and S. W. Gilbert, NAVSTAR: global positioning system - ten years later, Proceedings of the IEEE, pp. 1177-1186, 1983. 